\title{
Quantum Gravity Effects in Cosmology
}

\author{
Je-An Gu${ }^{1}$, Sang Pyo Kim ${ }^{2, \star}$, and Che-Min Shen ${ }^{3}$ \\ ${ }^{1}$ Leung Center for Cosmology and Particle Astrophysics, National Taiwan University, Taipei 10617, Taiwan \\ ${ }^{2}$ Department of Physics, Kunsan National University, Kunsan 54150, Korea \\ ${ }^{3}$ Department of Physics and Leung Center for Cosmology and Particle Astrophysics, National Taiwan Uni- \\ versity, Taipei 10617, Taiwan
}

\begin{abstract}
Within the geometrodynamic approach to quantum cosmology, we studied the quantum gravity effects in cosmology. The Gibbons-Hawking temperature is corrected by quantum gravity due to spacetime fluctuations and the power spectrum as well as any probe field will experience the effective temperature, a quantum gravity effect.
\end{abstract}

\section{Introduction}

Recent remarkable observations have made cosmology a science of precision. The more precise and accurate cosmological observations are, the more likely the possibility of quantum gravity effects are to be measured. In this talk, we review a quantum gravity effect in cosmological observations. There have been proposed different approaches to quantum gravity (for a review, see [1]). In partciular, the geometrodynamical approach to quantum gravity and cosmology seems to be practically useful in that the transition from quantum gravity to semiclasical gravity and then to classical gravity including quantum corrections for the Einstein and the energy-stress tensors prescribes dynamical laws and correlations in terms of classical cosmology variables and parameters [2].

The Wheeler-DeWitt (WDW) equation is the quantum law for the geometrodynamical approach to quantum gravity, which becomes a relativistic wave equation in the superspace of the scale factor $a$ and an inflaton $\phi$ for a Friedmann-Robertson-Walker universe. The scattering formulation of the two-component wave was advanced in Refs. [3, 4]. The Born-Oppenheimer interpretation of the WDW equation with respect to the Planck scale for gravity part and the energy scale for the inflaton separates the gravity part from the inflaton part and a further application of de Broglie-Bohm pilotwave theory results in the classical equation together with quantum corrections of gravitational and field fluctuations [5, 6] (for references, see [2]). The quantum cosmology for an inflationary model with inhomogeneous fluctuations predicted the power spectrum with quantum corrections, which is suppressed at large scales and provides a weaker upper bound on the Hubble constant $\mathrm{H}$ than the tensor-to-scalar ratio [7]. Other quantum gravity effects may be found in Ref. [8].

The main purpose of this proccedings' article is to compliment the recent discovery that the quantum cosmology results in an effective Gibbons-Hawking temperature due to quantum fluctuations [9]. To do this, we provide two new methods to find approximately the solution to the WDW equation for

\footnotetext{
^e-mail: sangkim@kunsan.ac.kr
} 
the FRW universe with a cosmological constant: the WKB approximation method and the LiouvilleGreen function method. From the wave function we can calculate the quantum potential, which gives rise to the quantum corrections to the semiclassical gravity. We then solve the semiclassical equation for the Eucliean geometry to find a periodic solution, whose period is the inverse temperature for the dS space in the sense of Ref. [10]. This approach may be an alternative to the gravitational instanton method [11]. Finally, we show that a probe field experiences this periodicity, which justifies the physical meaning of the temperature for dS space with quantum corrections.

\section{2 de Broglie-Bohm Pilot-Wave Theory for Quantum Cosmology}

The quantum cosmology based on the WDW equation leads to the semiclassical cosmology with quantum corrections as will be shown below. The WDW equation for a spatially closed FRW universe with a cosmological constant $\Lambda$, i.e, pure dS space, takes the form

$$
\left[-l_{p}^{2} \frac{\partial^{2}}{\partial a^{2}}+\frac{1}{l_{p}^{2}}\left(a^{2}-H_{\Lambda}^{2} a^{4}\right)\right] \Psi(a)=0,
$$

where $l_{p}=\sqrt{4 \pi G / 2}$ is the Planck length. Introducing a dimensionless variable $x=a / l_{p}$ and a constant $\bar{H}_{\Lambda}=H_{\Lambda} l_{p}$, the WDW equation describes a quantum problem with the zero energy for an inverted Mexican hat potential

$$
\left[-\frac{d^{2}}{d x^{2}}+x^{2}-\bar{H}_{\Lambda}^{2} x^{4}\right] \Psi(x)=0
$$

We now apply the de Broglie-Bohm pilot theory to Eq. (2) by expressing the solution in the oscillatory region as

$$
\Psi(x)=F(x) e^{i S(x)},
$$

where $F=|\Psi|$. Then, the real part of Eq. (2) becomes

$$
S^{\prime 2}-F^{\prime \prime}+x^{2}-\bar{H}_{\Lambda}^{2} x^{4}=0,
$$

and so does the imaginary part

$$
S^{\prime \prime} F+2 S^{\prime} F^{\prime}=0 .
$$

Substituting the solution $F=1 /|S|^{1 / 2}$ of Eq. (5) into Eqs. (3) and (4) is equivalent to the exact Wentzel-Kramers-Brillouin (WKB) solution or phase-integral formula. The essence of the de BroglieBohm pilot theory is to solve Eqs. (4) and (5) at the same time by regarding $F$ as a functional of $S$, i.e., $F(S)$. In the nonoscillatory region corresponding to a Euclidean spacetime, Eqs. (3)-(5) may be understood as an analytical continuation from the oscillatory region. In fact, introducing the cosmological time as the directional derivative of $x$ along the peak of the wave packet

$$
\frac{\partial}{\partial t}=-\frac{1}{x} \frac{\partial S}{\partial x} \frac{\partial}{\partial x}
$$

Eq. (4) becomes the semiclassical gravity equation $[5,6]$

$$
\left(\frac{\dot{x}}{x}\right)^{2}+\frac{1}{x^{2}}=\bar{H}_{\Lambda}^{2}-\frac{1}{x^{4}} V_{q}(x)
$$

where a dot denotes the derivative with respect to $t$ and $V_{q}=-F^{\prime \prime} / F$ is the quantum potential.

A passing remark is that Eq. (5) and thereby the quantum potential correspond to the flux (probability) conservation in the de Broglie-Bohm pilot theory of quantum mechanics [12]. On the other hand, the quantum potential provides a quantum correction in quantum cosmology to the classical gravity equation since there is no probability concept due to the single nature as a quantum system. The quantum potential is a consequence of spherical fluctuations of the FRW geometry. 


\section{Semiclassical Gravity Equation}

The quantum gravity effect in a pure gravity contributes the quantum potential to the semiclassical gravity as shown in Eq. (7). We may apply either the WKP approximation or the Liouville-Green (LG) transformation method, to the WDW equation (1) or (2). The LG transformation method, however, reduces to the WKB approximation in the limit of large variable while it also applies to the turning point up to an additional constant term [3].

From now on, we shall employ the LG transformation method, which rewrites Eq. (2) in terms of a new variable as [3]

$$
\left[\frac{\partial^{2}}{\partial \eta^{2}}-\left(\eta^{m}+\Delta(\eta)\right)\right] \tilde{\Psi}(\eta)=0
$$

where

$$
\left(\frac{\partial \eta}{\partial x}\right)^{2} \eta^{m}=V_{g}(x), \quad \Delta(\eta)=-\frac{3}{4} \frac{\left(\frac{d^{2} \eta}{d x^{2}}\right)^{2}}{\left(\frac{d \eta}{d x}\right)^{4}}+\frac{1}{2} \frac{\left(\frac{d^{3} \eta}{d x^{3}}\right)^{2}}{\left(\frac{d \eta}{d x}\right)^{3}} .
$$

and

$$
\tilde{\Psi}=\sqrt{\frac{d \eta}{d x}} \Psi(x), \quad V_{g}(x)=x^{2}-\bar{H}_{\Lambda}^{2} x^{4} .
$$

Note that $\Delta(\eta)$ is proportional to the Schwazian derivative as $\Delta(\eta)=\left(1 / 2(d \eta / d x)^{2}\right)(S \eta)(x)$. The degree $m$ is chosen by the order of roots of $V_{g}(x)=0$. The classical motion is allowed in the region outside the simple root $x_{H}=1 / \bar{H}_{\Lambda}$, so we choose $m=1$.

For large $x$, i.e., the scale factor $a$ bigger than the Hubble radius $1 / \bar{H}_{\Lambda}$ in the Planckian unit, $\Delta(\eta) \approx 1 / \eta^{2}$. In this asymptotic region, we can neglect $\Delta(\eta)$ and solve Eq. (8) in terms of the Hankel functions as

$$
\Psi_{\exp }(x)=D_{1} \frac{\xi^{1 / 2}}{V_{g}^{1 / 4}} H_{1 / 3}^{(2)}(\xi), \quad \Psi_{\text {con }}(x)=D_{2} \frac{\xi^{1 / 2}}{V_{g}^{1 / 4}} H_{1 / 3}^{(1)}(\xi),
$$

where $D$ 's are integration constants and

$$
\xi=i \int^{x} \sqrt{V_{g}\left(x^{\prime}\right)} d x^{\prime}
$$

Here, $\Psi_{\exp }(x)$ corresponds to an expanding branch of the wave function while $\Psi_{\text {con }}(x)$ corresponds to a contracting branch. In the limit of large $x$, which belongs to the WKB region,

$$
\xi=-\int^{x} \sqrt{\bar{H}_{\Lambda}^{2} x^{\prime 4}-x^{\prime 2}} d x^{\prime}=-S(x)
$$

the asymptotic form of the Hankel function

$$
H_{1 / 3}^{(1)}(\xi)=\sqrt{\frac{2}{\pi \xi}} e^{i\left(\xi-\frac{5 \pi}{12}\right)}, \quad H_{1 / 3}^{(2)}(\eta)=\sqrt{\frac{2}{\pi \xi}} e^{-i\left(\xi-\frac{5 \pi}{12}\right)}
$$

gives the WKB approximation, up to a constant phase,

$$
\Psi_{\text {exp }}(x)=D_{1} \frac{1}{\left|V_{g}\right|^{1 / 4}} e^{i\left(S(x)-\frac{5 \pi}{12}\right)}, \quad \Psi_{\operatorname{con}}(x)=D_{2} \frac{1}{\left|V_{g}\right|^{1 / 4}} e^{-i\left(S(x)-\frac{5 \pi}{12}\right)} .
$$

Including correction (9) at the turning point, a straightforward calculation approximately leads to the quantum potential for large and small $x$ as

$$
V_{q}(x)=-\frac{2}{x^{2}} \quad\left(x \gg \frac{1}{\bar{H}_{\Lambda}}\right), \quad V_{q}(x)=-\frac{3}{4 x^{2}} \quad\left(x \leq \frac{1}{\bar{H}_{\Lambda}}\right) .
$$




\section{Gibbons-Hawking (GH) Temperature corrected by QC}

The semiclassical gravity equation (7) now reads

$$
\left(\frac{\dot{a}}{a}\right)^{2}+\frac{1}{a^{2}}=H_{\Lambda}^{2}+\frac{c\left(l_{p}\right)^{4}}{a^{6}}
$$

which is Eq. (16) of Ref. [9]. Here, $c=2$ for large $a$ and $c=3 / 4$ for small $a$. Note that a homogeneous massless field in the FRW geometry contributes the same form as the spherical fluctuations of the geometry [13]. The classical gravity has the solution $a(t)=\cosh \left(H_{\Lambda} t\right) / H_{\Lambda}$, which has the Euclidean solution $a(\tau)=\cos \left(H_{\Lambda} \tau\right) / H_{\Lambda}$ in the classically forbidden region. The Gibbons-Hawking temperature is the inverse of the periodicity $T_{\mathrm{GH}}=H_{\Lambda} /(2 \pi)$ as shown in Ref. [10]. A probe field in the dS space experiences this periodicity, whose correlation function exhibits the Bose-Einstein or Fermi-Dirac distribution with the Gibbons-Hawking temperature.

We now solve Eq. (17) in the Euclidean time $\tau=i t$ in the Euclidean spacetime by the quadrature method, which leads to the periodicity

$$
\tau=2 \int_{a_{-}}^{a_{+}} \frac{d a}{\sqrt{1-H_{\Lambda}^{2} a^{2}-\frac{3\left(l_{p}\right)^{4}}{4 a^{4}}}}=\frac{2}{H_{\Lambda}} \int_{u_{-}}^{u_{+}} \sqrt{\frac{u}{\frac{u^{2}}{H_{\Lambda}^{2}}-u^{3}-\frac{3\left(l_{p}\right)^{4}}{4 H_{\Lambda}^{2}}}} d u,
$$

where $u=a^{2}$ and another factor of two is multiplied in the second line in order to include the motion in the region of $a \leq 0$. Equation (18) is the complete elliptic integral of third kind [14]

$$
\tau=\frac{2}{H_{\Lambda}} \int_{u_{-}}^{u_{+}} \sqrt{\frac{u}{\left(u_{+}-u\right)\left(u-u_{-}\right)\left(u-u_{0}\right)}} d u,
$$

where $u_{ \pm}$are two positive roots of the cubic equation of $u$ in Eq. (18). We may perturbatively find the roots as

$$
u_{+}=\frac{1}{H_{\Lambda}^{2}}-\frac{3}{4}\left(H_{\Lambda} l_{p}\right)^{2}, \quad u_{-}=\frac{\sqrt{3}}{2} l_{p}^{2}
$$

and, by neglecting the term $l_{p}^{4} / H_{\Lambda}^{2}=\left(R_{H} l_{p}\right)^{2} l_{p}^{2}$ with the Hubble radius $R_{H}=1 / H_{\Lambda}$, integrate approximately as

$$
\tau_{q} \approx \frac{2}{H_{\Lambda}}\left(\arcsin \left(1-\frac{3}{2}\left(H_{\Lambda} l_{p}\right)^{4}\right)+\arcsin \left(1-\sqrt{3}\left(H_{\Lambda} l_{p}\right)^{2}\right)\right)
$$

Thus, the temperature from the inverse periodicity is approximately given by

$$
T_{q}=\frac{H_{\Lambda}}{2 \pi}\left(1+\frac{(12)^{1 / 4}}{\pi} H_{\Lambda} l_{p}\right)
$$

and confirms the result from Ref. [9]. The quantum correction to the Gibbons-Hawking temperature is the order of $H_{\Lambda} l_{p}$.

\section{Conclusion}

In this paper, we have studied the quantum effect of spherical fluctuations of spacetime to the GibbonsHawking temperature. For this purpose, we have employed the semiclassical gravity theory based on the WDW equation and provided the semiclassical gravity for a pure gravity with the LG transformation method to calculate the quantum potential. The LG transformation method reduces to the WKB 
approximation in the limit of large variable. The LG transformation method approximately gives the wave function itself in terms the Hankel functions, from which the amplitude and thereby the quantum potential can be analytically derived. We noted that both the methods confirm the Gibbons-Hawking temperature corrected by quantum gravity to be consistent [9].

We now provide a physical reasoning why a probe field could measure the effective GibbonsHawking temperature in this paper. A homogeneous probe field $\phi$ of a light mass $m$ does not give a back-reaction enough to change the spacetime itself governed by the semiclassical gravity and obeys the equation of motion in the Cauchy formulation of the first order:

$$
\frac{d}{d t}\left(\begin{array}{c}
\phi \\
\dot{\phi}
\end{array}\right)=\left(\begin{array}{cc}
0 & 1 \\
-m^{2} & -3 \frac{\dot{a}}{a}
\end{array}\right)\left(\begin{array}{l}
\phi \\
\dot{\phi}
\end{array}\right) .
$$

The Floquet theory guarantees the periodicity for the probe field and its correlation function due to the periodicity of the semiclassical gravity (17) for the FRW universe. Thus, the semiclassical gravity goes beyond the classical FRW geometry with a stationary Killing vector but preserves a similar property. The Gibbons-Hawking temperature corrected by quantum gravity is a physical one to be measured by the probe field [15] and seems to be universal. The power spectrum of inflation models is similarly expected to have the quantum gravity effect due to the effective temperature.

\section{Acknowledgements}

S. P. K. would like to thank Rong-Gen Cai, Jorge Armando Rueda Hernández, Remo Ruffini and Gregory Vereshchagin for useful discussions during ICGAC13-IK15 and Maurice van Putten for the question of radiation for the Gibbons-Hawking temperature. This work was supported by the Basic Science Research Program through the National Research Foundation of Korea (NRF) funded by the Ministry of Education (NRF-2015R1D1A1A01060626).

\section{References}

[1] Approaches to Fundamental Physics, edited by I-O. Stamatescu and E. Seiler, Lect. Note Phys. 721 (Springer-Verlag, Berlin, 2007).

[2] S. P. Kim and W. Kim, Int. J. Mod. Phys. Conf. Ser. 43, 1660205 (2016) [arXiv:1603.08087].

[3] S. P. Kim, Phys. Rev. D 46, 3403 (1992).

[4] S. P. Kim, The Universe 1, no. 1, 11 (2013) [arXiv:1304.7439].

[5] S. P. Kim, Phys. Rev. D 52, 3382 (1995) [gr-qc/9511038].

[6] S. P. Kim, Phys. Rev. D 55, 7511 (1997) [gr-qc/9611040].

[7] C. Kiefer and M. Kraemer, Phys. Rev. Lett. 108, 021301 (2012) [arXiv:1103.4967].

[8] S. Carlip, D. W. Chiou, W. T. Ni and R. Woodard, Int. J. Mod. Phys. D 24, 1530028 (2015) [arXiv:1507.08194].

[9] J. A. Gu, S. P. Kim and C. M. Shen, Int. J. Mod. Phys. D 26, 1750116 (2017).

[10] R. Brout, Found. Phys. 17, 603 (1987).

[11] S. W.Hawking and I. G. Moss, Phys. Lett. B 110, 35 (1983).

[12] I. Licata, D. Fiscaletti, Quantum potential: Physics, geometry and algebra, (Springer, Heidelberg, 2014).

[13] S. P. Kim, Phys. Lett. A 236, 11 (1997) [gr-qc/9703065].

[14] I. S. Gradshteyn and I. M. Ryzhik, Table of Integrals, Series, and Products, (Academic Press, London, 1994).

[15] B. .Kay and R. M. Wald, Phys. Rep. 207, 49 (1991). 